\section{Photoshop Distilled: Basic Black and White Image Adjustment for Microscopists \\ Tina (Weatherby) Carvalho,}

Biological EM Facility, University of Hawaii

Ready or not, digital imaging in the microscope lab is here to stay. While I am one of those fogies who insist that digital images are nowhere near as good as photographic prints, I've also bowed to the inevitable, and am beginning to find ways to assist the clients in our multi-user facility with their digital imaging needs. We can acquire digital images from our FESEM, from our light microscope with a CCD, and from a flatbed scanner. Clients use their images for image analysis, transport to others scientists via the Internet or World Wide Web, for reports, slides, overhead transparencies, and publication. Generally, these images need to be manipulated for use, just as one would manipulate a photographic print to correct any deficiencies in exposure or area of view. Adobe PhotoShop is the software of choice for image adjustment, accounting for more than $80 \%$ of the digital image-editing software market. Photoshop is extremely powerful, and the ethics of using it to manipulate visual data is being hotly debated. I strongly feel that any manipulation beyond what is outlined here would be unacceptable, and I'm sure there are some who will feel that even this is too much, but that is the subject for another article! The learning curve for Photoshop is steep, and the majority of the available books deal at length with color management and effects. We, as scientists, however are more interested in faithful reproduction and presentation of our visual data. To this end, l've tried to distill the contents of many books and a two-day workshop down to the basic techniques of interest to microscopists. The following is a step-by-step minitutorial designed to lead you through the rudiments of Photoshop, taken from a manuscript I am preparing, tentatively titled "Photoshop Distilled: Basic

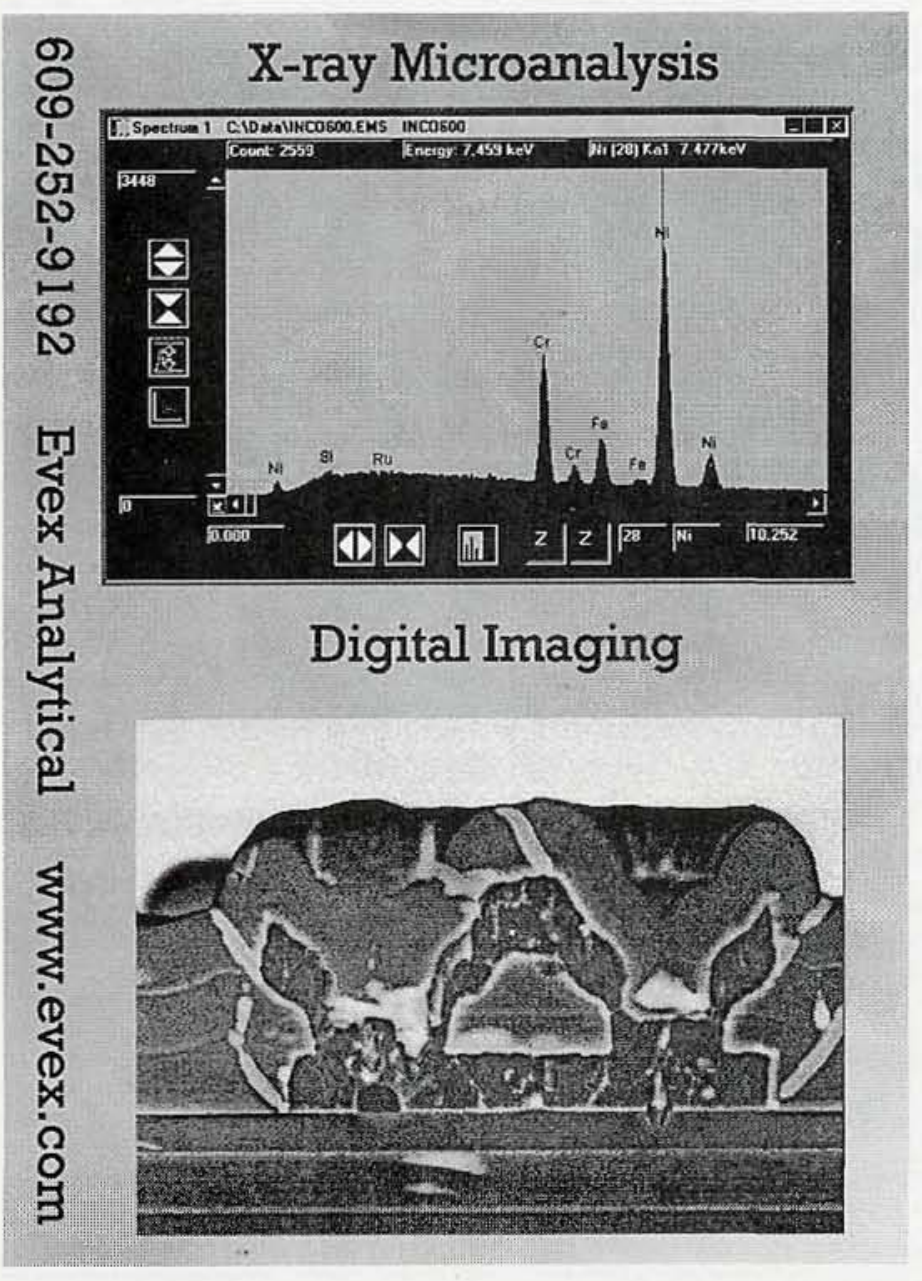

Black and White Image Adjustment and Layout for Scientists". If you have any corrections, comments, or contributions, please feel free to e-mail me at tina@pbrc.hawaii.edu.

\section{Image Adjustment}

1. Start up Adobe Photoshop 3.0 or 4.0 .

2. Select File $\rightarrow$ Acquire to obtain an image with a scanner or other plug-in, or:

3. Select File $\rightarrow$ Open, then find and select the filename for an existing image, such as one obtained with NIH Image. If your file doesn't have an extension recognizable to Photoshop, click on Show All Files to find and then rename it.

4. Crop the image if necessary.

- In 3.0 or 4.0 you may discard a few pixels around an image by selecting Image $\rightarrow$ Canvas Size, selecting a slightly smaller size than your image, and then placing your image in the center or to one side.

- For more cropping, use the Crop Tool. In 4.0 press $C$ to select it or hold the mouse button down over the rectangle selection tool in the Toolbox to get a pop-up menu of other selection tools, and select the Crop Tool. In 3.0 click on the Crop Tool in the toolbox.

- Drag the crop marquee around the portion of the image you want to keep, then double-click inside it (4.0) or click once with the scissors cursor (3.0) to crop. - In 4.0 the Crop Tool may also be resized and rotated before cropping. In 3.0 you must Select All of the image, then use Image $\rightarrow$

5. Adjust image tones:

- Select Image $\rightarrow$ Adjust $\rightarrow$ Levels and get the gray levels histogram

- Adjust input levels:

- Move the left, black arrow under the histogram to the right to select the darkest input pixels, or enter a value in the leftmost Input Leveis option box (e.g., 20).

- Move the right, white arrow to the left to select the lightest pixels, or enter a value in the rightmost option box (e.g., 235).

- For midtone, or gamma correction, move the middle, gray arrow to the left, or enter a value in the middle option box (e.g., 1.8). Be aware that in order to get a good print you will need to adjust your on-screen image until it shows the full range of grays but overall looks too light! This is due to the fact that the image you see on your monitor has already had gamma values applied to it that will not be sent to your printer.

- Click on OK. Check image, and re-do gamma correction again, if needed. 6. Determine if image needs sharpening. Clleck your ethics barometer; will this change your data?!

- View image in the size it will be printed (in 4.0 by selecting View $\rightarrow$ Print Size). Resampling or resizing up (not a good idea) or down changes the apparent sharpness. Best sharpening is accomplished by Filter $\rightarrow$ Unsharp Mask:

- Set a value in the dialog box (e.g., 144\%). Values less than about 25 will be subtle and more than about 300 will be too dramatic.

- Select a radius of perhaps 1 to 4 pixels.

- Select a threshold of perhaps 0 to 16 levels.

- Click OK.

7. Save your file using Save As... and select a name, format and location for it. - For greatest fidelity and transferability between computer platforms, select TIFF with no compression.

- TIFF with LZW compression will compress the file size somewhat with no loss of information, but may not be accepted by some computers.

- The best compression is performed by selecting JPEG and then the highest level of $10(4.0)$ or maximum setting (3.0). JPEG is a lossy compression scheme, meaning that some information is lost, but often this loss is unnoticeable.

\section{Applying Magnification Bars}

Applying a magnification bar in Photoshop makes sense because then it is always available with your image, no matter what its ultimate fate, such as reduction in print or as a slide. Other labeling with text and arrows can be done 
either with Photoshop (the subject of the next section) or, perhaps more effectively, with additional programs such as PowerPoint, PageMaker, Quark Express, etc.

1. With your image file open, go to Window $\rightarrow$ Show Rulers. You may set the units to centimeters, inches, or picas in File $\rightarrow$ Preferences $\rightarrow$ Units.

2. Select the color you want your bar as your foreground color. To get true black or white, click on the tiny black and white rectangles at the bottom of the Toolbox; black will be the foreground color. White can be brought to the foreground by clicking on the curved arrow pointing between the two.

3. Double-click on the Line Tool to get the options palette. Select Antialiased, Normal, and $100 \%$ opacity. Enter a line width (e.g., 4 pixels) and make sure that Arrowheads Start or End are not selected.

4. Use the Line Tool cursor lines at the top and side rulers to position and measure the length of the line that you draw by pressing the mouse button and dragging on your image.

5. If you made a mistake or don't like your line, use Edit $\rightarrow$ Undo before you do anything else.

6. Make sure you remember what this magnification bar represents. You may append notes to the image by selecting File $\rightarrow$ File Info. This information will be available to you each time you open the image.

\section{Labeling images in Photoshop}

Addition of type to digital images is best done with software designed for that purpose, such as QuarkExpress, Adobe PageMaker, or Microsoft PowerPoint. With Photoshop, type is treated as another bitmapped image and, as such, will exhibit pixelation at low resolutions. However, at high resolutions the jaggedness is not so apparent. Check the anti-aliasing box in the Text options palette if you wish to have PhotoShop soften the edges of the type. Type applied to an image will have the same resolution as that image.

1. To apply text to an image, click on the Type Tool (T) in the Toolbox. Your cursor will become an I-beam. Position this cursor over the image approximately where you would like the type and click. You will get a dialog box.

- Select Font, Size, Style(s) and Alignment.

- Type your label in the box, then click OK.

- Your text will appear in the foreground color and as a selection with marching ants (3.0) or as its own layer (4.0). To turn of the marching ants in 3.0 so that you can more readily see the text, select Select $\rightarrow$ Hide Edges, but note that it still remains a selection until you click elsewhere or otherwise deselect it.

-While selected or in its own layer, text can be moved with the Move Tocl or with the keyboard arrows. If you make a mistake, the type, while still selected, is in its own layer (4.0) or as a floating layer (3.0) and can still be deleted with the delete key (3.0) or thrown in the trash in the layers palette $(3.0$ and 4.0$)$.

- Deselect to paste the label down in 3.0. If this is a mistake, you can Edit $\rightarrow$ Undo if it is the last thing you did. In 4.0 you can delete this layer now or later.

- In 4.0 text always appears as a new layer. Non-text additions, such as arrows, cannot be applied to text layers.

2. To apply arrows to an image, double-click on the Line Tool in the Toolbox to see the Line Tool Options palette.

- Select Line Width in pixels (e.g., 4), Anti-aliasing, Normal, and $100 \%$ Opacity.

- Select Arrowheads Start or End, depending on whether you want the arrowhead to start where you begin to drag your cursor, or end where you stop your cursor.

- Select Shape $\rightarrow$ Width (e.g., 400\%), Length (e.g., 600\%), and Concavity (e.g., 16\%), the amounts of which are related to the Line Width. Click on OK.

- The arrow will be in your foreground color, so check it now.

- Your best bet is to place arrows in their own layer. Go to Window $\rightarrow$ Show Layers palette and click on the little folded paper icon at the bottom, or to the right-pointing black triangle on the top right and select New Layer from the pop-up menu. This also works for 3.0 .

- Drag the mouse across your image to make an arrow.
- In 3.0 you may hide the marching ants but maintain the selection by going to Select $\rightarrow$ Hide Edges, allowing you to better position the arrow.

- In 3.0 you may move the arrow around with the Move Tool or keyboard arrows. In 4.0 you must use the Move Tool, and it will move the entire layer.

- If you don't like your arrow, go to Edit $\rightarrow$ Undo while it's still the last thing you did $(3.0,4.0)$ or throw away the layer $(4.0)$.

- Save As a new filename so that the labeled image stays separate from your unlabeled one. Trust me, you may want your unlabeled image back someday!

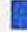

\section{Applications/Software Specialist}

American Nuclear Systems, Inc. has an immediate opening for a senior applications specialist. The successful candidate will have experience in scanning electron $\mathrm{mi}$ croscopy and quantitative $\mathrm{x}$-ray microanalysis. A strong theoretical background in quantitative analysis techniques is essential. This person will be in charge of our new applications laboratory. $\mathrm{He} /$ she will interface with our software development group in the development of analytical techniques as well as supporting customers with their analytical problems.

ANS offers a full range of benefits and an exciting key contributor compensation plan. Interested parties should contact Bill Hardy, in confidence, at (423)482-1665 or eMail at bhardy@ansxray.com

\section{.}

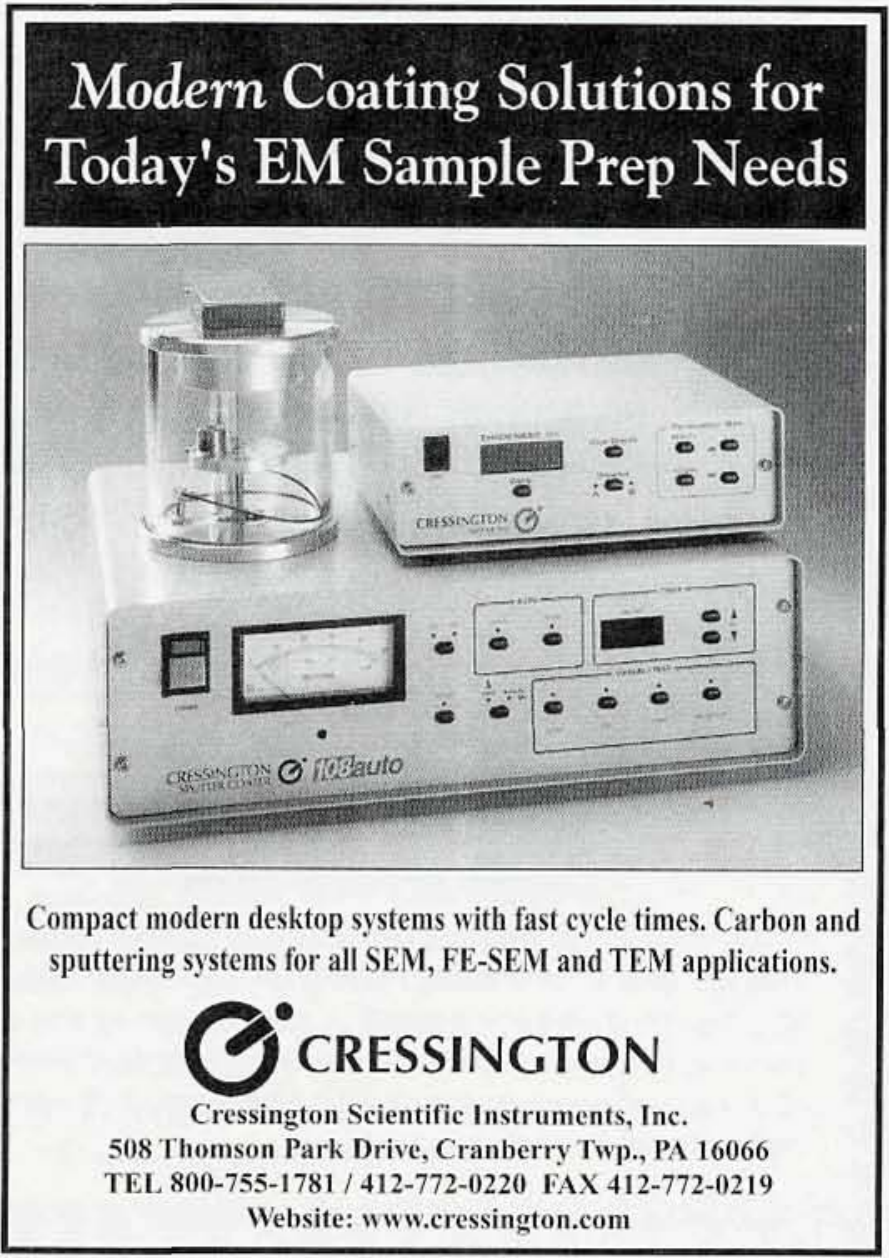

